\title{
Relationship between fragmentation, degradation and native and exotic species richness in an Andean temperate forest of Chile
}

\section{Relación entre fragmentación, degradación y riqueza de especies nativas y exóticas en un bosque templado andino de Chile}

\author{
Isabel Rojas ${ }^{1 *}$, Pablo Becerra ${ }^{2}$, Nicolás Gálvez ${ }^{1,4}$, Jerry Laker ${ }^{1,3}$, Cristián Bonacic ${ }^{1,2}$ \& Alison \\ HESTER ${ }^{3}$ \\ ${ }^{1}$ Laboratorio Fauna Australis, Departamento de Ecosistemas y Medio Ambiente, ${ }^{2}$ Departamento de Ecosistemas y Medio \\ Ambiente, Pontificia Universidad Católica de Chile, Av. Vicuña Mackenna 4860, Santiago, Chile. \\ ${ }^{3}$ Macaulay Land Use Research Institute, Craigiebuckler, Aberdeen AB15 8QH, UK. \\ ${ }^{4}$ Departamento de Ciencias Naturales, Centro de Desarrollo Local (CEDEL), Pontificia Universidad Católica de Chile, O’Higgins \\ 501, Villarrica, Chile. \\ *imrojas@uc.cl
}

\begin{abstract}
Human impact such as forest fragmentation and degradation may have strong effects on native and exotic plant communities. In addition, these human-caused disturbances occur mostly in lowlands producing greater fragmentation and degradation there than in higher elevations. Plant invasion should be greater in more fragmented and degraded forests and hence lowlands should be more invaded than higher elevations. In turn, native species richness should be negatively related to fragmentation and degradation and hence greater in higher elevations within a forest type or elevation belt. We assessed these hypotheses in an Andean temperate forest of southern Chile, Araucanía Region. We recorded the vascular plant composition in twelve fragments of different size, perimeter/area, elevation level and evidence of human degradation (logging, fire, cattle faeces). Based on these variables we performed a fragmentation and a degradation index. Pearson correlations were used to analyze the relationship between all these variables. We found that fragmentation and degradation were positively correlated, and each of them decreased with altitude. Furthermore, fragmentation and degradation affected native and exotic species richness in different ways. Invasion was enhanced by both fragmentation and degradation, and as consequence of the altitudinal patterns of these human-caused disturbances, invasion seems to occur mainly in lowlands. In turn, native species richness decreased with fragmentation, and it was not related to degradation nor altitude.
\end{abstract}

KEYWORDS: Fragmentation, forest degradation, elevation gradient, invasion, plant diversity.

\section{RESUMEN}

Impactos humanos tales como la fragmentación y degradación de bosques pueden tener fuertes efectos en las comunidades de especies vegetales nativas y exóticas. Además, perturbaciones antrópicas ocurren principalmente en menores altitudes produciendo mayores grados de fragmentación y degradación que en mayores altitudes. La invasión de plantas exóticas debería ser mayor en bosques más fragmentados o degradados y, por lo tanto, en menores altitudes dentro de un tipo de bosque o piso altitudinal. En cambio, la riqueza de especies nativas debería ser negativamente afectada por la fragmentación y degradación, encontrándose mayor riqueza en mayores altitudes dentro de un tipo de bosque determinado. En este trabajo evaluamos estas hipótesis en un bosque templado andino de la Región de la Araucanía, Chile. Registramos la composición de plantas vasculares en doce fragmentos de diferente tamaño, razón perímetro/área, altitud y degradación antrópica (cortas, incendios, fecas de ganado). En base a estas variables construimos un índice de fragmentación y uno de degradación para estos fragmentos. Se analizaron las relaciones entre estas variables a través de correlaciones de Pearson. Nuestros resultados sugieren que la fragmentación y degradación están positivamente relacionadas y que ambos tipos de perturbación ocurren en altitudes más bajas del tipo de bosque estudiado. Además, la fragmentación y degradación están afectando en diferente forma a la riqueza de especies nativas y exóticas. La invasión se incrementó como consecuencia tanto de fragmentación como de degradación, y como consecuencia del patrón de distribución altitudinal de estas perturbaciones, la invasión aparentemente ocurre principalmente en zonas bajas. En cambio, la riqueza de especies nativas fue negativamente afectada sólo por la fragmentación, y no se relacionó con la degradación interna de los bosques ni con la altitud.

Palabras clave: Fragmentación, degradación, gradiente altitudinal, invasión, diversidad vegetal. 


\section{INTRODUCTION}

The temperate forests of southern South America are recognized as a global biodiversity hotspot because of their endemic and endangered biota (Myers et al. 2000). However, the processes of colonization for agriculture and cattle ranching have strongly degraded and fragmented these forests (Armesto et al. 1998). These processes of habitat degradation and fragmentation are amongst the top five current threats to biodiversity in Chile (Ceballos et al. 2009) as well as globally (Farhig 2003, Fischer \& Lindenmayer 2007).

With fragmentation, habitat availability and the area available to native species of the original ecosystems are limited to smaller, often disconnected patches within a matrix of other new habitats (Wilcove et al. 1986, Saunders et al. 1991, Fischer \& Lindenmayer 2007). On the other hand, habitat degradation implies both loss of quality and structural changes, which may be caused by human activities such as clearance, selective logging, cattle grazing, and fires (Lindenmayer \& Fischer 2006). Though degradation may occur both in fragmented and continuous habitats, a relationship between fragmentation and degradation has previously been documented; fires, selective logging and cattle have been found more frequently in smaller fragments (Cochrane 2001, Hobbs 2001, Jaña et al. 2007).

Degradation and fragmentation have been frequently related to different changes in plant communities, in particular, declining native species diversity (e.g. Saunders et al. 1991, Soulé et al. 1992, Gilliam et al. 1995, Laurance et al. 1998, Tabarelli et al. 1999, Hersperger \& Forman 2003, Lindenmayer \& Fischer 2006, Echeverría et al. 2007, Schmitt et al. 2010), as well as increases in exotic species diversity and abundance (Brothers \& Spingarn 1992, Hobbs \& Huennecke 1992, Wilson et al. 1992, D’Antonio 1993, Mooney \& Hobbs 2000, Hobbs 2001, Rouget et al. 2002, Pauchard \& Alaback 2004, Sax et al. 2005). These fragmentation effects may be related to micro-environmental changes in the new edges, such as increase of light, higher temperatures and the potential release of other resources (Saunders et al. 1991, Brothers \& Spingarn 1992, Chen et al. 1995, Hobbs 2001). In addition, Brothers \& Spingarn (1992) proposed that decrease in native species in fragmented ecosystems may reduce biotic resistance against further invasion. However, this is clearly not always the case, and there are studies where no invasion has been observed in remnant patches of habitat that keep their internal structure (Teo et al. 2003). Similarly, habitat degradation has also been documented as a facilitator of exotic invasion. For example, events causing canopy and soil openings may create microenvironmental conditions that stimulate germination and establishment of invasive species (Sakai et al. 2001). This may also adversely affect native species, especially as exotic species can be more competitive than native species under disturbed conditions, thus further favouring invasion (Brothers \& Spingarn 1992, Hester \& Hobbs 1992, Hobbs \& Huennecke 1992, Sakai et al. 2001, Teo et al. 2003). Furthermore, large herbivores (cows, horses, swine, etc.) can also be significant seed vectors of exotic species, and cause large scale modifications in understory vegetation, adversely affecting native species and favouring invasion (Hobbs 2001, Vázquez 2002).

On the other hand, human activity tends to concentrate in sites that are environmentally more benign and easier to access, having for instance better weather, deeper and more fertile soils, generally located in lower altitudes. This may produce a non-random distribution pattern of fragmentation, degradation, native diversity and plant invasion (Hobbs 2001, Lindenmayer \& Fischer 2006, Alexander et al. 2011). Lower altitude sites, where more productive habitats are found, have customarily been used for farming, logging and urban development, resulting in smaller remnant fragments that are more degraded and invaded by alien species than those in higher elevations (Pauchard \& Alaback 2004, Schmitt et al. 2010, Alexander et al. 2011).

In different Chilean temperate forests, previous studies have documented several negative impacts of forest fragmentation and degradation on native biodiversity (Bustamante et al. 2005, Grez et al. 2006, Echeverría et al. 2007). However, little is known about the effects of these human-caused disturbances on plant invasion. Some studies performed in Chile have assessed the impact of fragmentation (Bustamante \& Simonetti 2005, Bustamante et al. 2003) and forest degradation (e.g. Pauchard \& Alaback 2004, Becerra 2006, Fuentes-Ramírez et al. 2010) on plant invasion. Also, some biogeographical studies have correlated exotic species richness with human settlement documenting greater plant invasion where mostly forest fragmentation and degradation has occurred-mainly in the centre-south region (Arroyo et al. 2000, Castro et al. 2005). However, in Chile no study has assessed the relationship between fragmentation, degradation and altitude and their effects on plant invasion. In this paper we examined how exotic and native species richness are correlated with fragmentation and degradation along an altitudinal gradient in an andean temperate forest. We expect that fragmentation and degradation will affect positively the exotic species and negatively the native species richness. We also expect that fragmentation and degradation will be greater in lower altitudinal levels and hence that exotic species richness should be negatively and native richness positively related to altitude. As consequence of these trends, we also expect that native and exotic species richness will be negatively related. 


\section{METHODS}

\section{STUDY AREA}

We selected 12 forest fragments (Table I) on private land estates in the pre-Andean zone of the Araucanía Region, specifically in Cautín, Pucón and Curarrehue districts $\left(39^{\circ}\right.$ $\mathrm{S}, 72^{\circ} \mathrm{W}$ ) (Fig. 1). The mean altitude of these fragments (average between the higher and lower level of each fragment) varied between 300 and 1020 m a.s.l. (Table I). The area has deep andisoils or fine volcanic soils with good water-retention capacity and is well-drained (Pauchard \& Alaback 2004). The climate in this area is temperate-humid, with a short dry season (less than 4 months) and 2,000 mm annual average rainfall, predominantly in winter (Di Castri $\&$ Hajek 1976). Maximum and minimum mean temperatures for the warmest month (January) are $25.3^{\circ}$ and $10.4^{\circ} \mathrm{C}$ respectively, while in the coldest month (July), maximum and minimum mean temperatures range from $12.1^{\circ} \mathrm{C}$ to 4. $2^{\circ} \mathrm{C}$ (Di Castri \& Hajek 1976).

The natural vegetation of the study area is comprised mostly by one type of forest corresponding to a mix of deciduous species such as Nothofagus alpina (Poepp. \& Endl.) Oerst., Nothofagus obliqua (Mirb.) Oerst., and evergreen species such as Persea lingue (Miers ex Bertero) Nees, Aextoxicon punctatum Ruiz \& Pav., Laurelia sempervirens (Ruiz \& Pav.) Tul., Nothofagus dombeyi (Mirb.) Oerst., Laureliopsis philippiana (Looser) Schodde, and Saxegothaea conspicua Lindl. (Gajardo 1993). The matrix surrounding fragmented patches is composed by grasslands for cattle farming, croplands, and scrub and forest plantations in almost all fragments.
VEGETATION SURVEY

In the summer of 2008 , all patches were sampled to gather information on floristic plant composition and anthropic indicators. Fragmentation level was evaluated by the patch size and perimeter/area ratio. Both variables were determined from orthorectified aerial photographs (2007) using the software Arc-View 3.2. Two $140 \mathrm{~m}$-long linear transects were sampled in each patch $100 \mathrm{~m}$ apart. Transects were located in an altitudinal range between 300 and $900 \mathrm{~m}$ a.s.l. (including all patches) (Table I), as close as possible to the centre of the patches. In each transect, eight $25-\mathrm{m}^{2}$ $(5 \times 5 \mathrm{~m})$ plots were located every $15 \mathrm{~m}$ along both lines. The number and composition of vascular plants (excluding epiphyte species) was recorded for each plot, determining the biogeographical origin (native or exotic) and life form of each species. Nomenclature follows The International Plant Names Index (http://www.ipni.org/index.html). Stumps from logging, livestock faeces and fire-signs were also counted in each $5 \times 5 \mathrm{~m}$ plot. First, we added values of each variable among all plots and transects per fragment to obtain one value per fragment. Then, separately by variable, stump, faeces and fire-sign numbers were indexed from 0 to 1 through the division of their counts per transect by the maximum number recorded among all fragments. These rates were used to build a degradation index by adding values of these three factors. Degradation indexes therefore vary from 0 to 3 , where 0 indicates no degradation and 3 is the maximum degradation shown by the assessed variables. In the same way, the patch size and the perimeter/area ratio (one value per fragment) were indexed from 0 to 1 separately through the division of their counts per fragment

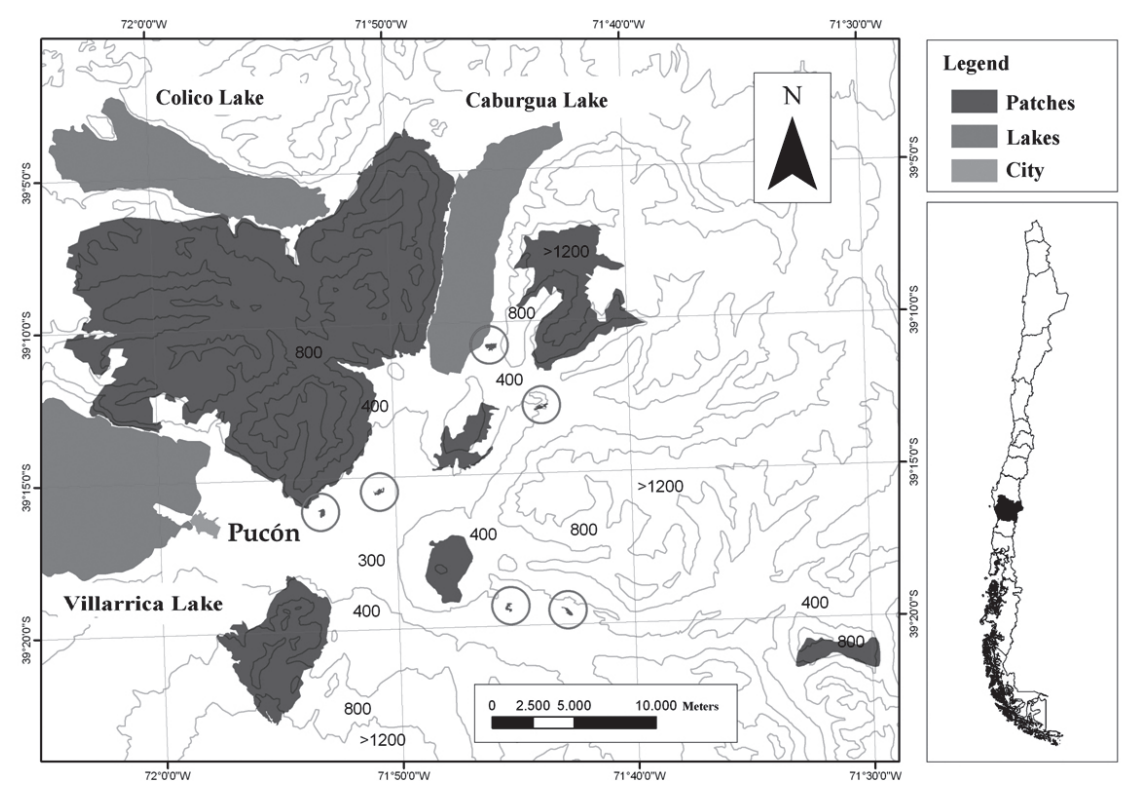

Figure 1. Study Area. Location in Chile and studied fragments are shown. Circles show smaller fragments.

Figura 1. Area de estudio. Se muestra la ubicación en Chile y los fragmentos estudiados. Círculos muestran los fragmentos más pequeños. 
Gayana Bot. 68(2), 2011

TABLE I. Fragments included in the study. Altitudinal range, altitude of transects in each fragment and area of fragments. Smaller patches are enumerated from $\mathrm{P} 1$ to $\mathrm{P} 6$.

TABLA I. Fragmentos incluidos en el estudio. Rango altitudinal, altitud de transectos muestreados en cada fragmento y área de fragmentos. Fragmentos más pequeños son enumerados como P1 a P6.

\begin{tabular}{lccc}
\hline Fragment name & Altitude range (m a.s.l.) & Transect altitude & Area (ha) \\
\hline Kawelluco & $447-1207$ & 768 & 3,242 \\
Namuncai & $661-1276$ & 760 & 29,642 \\
La Barda & $517-1160$ & 700 & 729 \\
Río Nevado & $630-1400$ & 690 & 3,253 \\
Curarrehue & $560-1260$ & 860 & 598 \\
Huelemolle & $610-843$ & 775 & 805 \\
P 1 & $562-711$ & 640 & 21 \\
P 2 & $404-442$ & 430 & 16 \\
P 3 & $370-378$ & 375 & 12 \\
P 4 & $300-352$ & 315 & 14 \\
P 5 & $269-333$ & 320 & 13.5 \\
P 6 & $384-411$ & 395 & 13 \\
\hline
\end{tabular}

by the maximum number recorded among fragments. These rates were used to build a fragmentation index by adding values of these two factors. Fragmentation indexes can vary between 0 and 2, being 0 the minimal fragmentation and 2 the maximal fragmentation.

An overall number of native and exotic species per fragment was derived from combining the total observed species lists from all $5 \times 5 \mathrm{~m}$ plots and both transects per fragment.

\section{Statistical ANALYSES}

We first assessed normality distribution of data (Shapiro Wilk's test: Degradation Index, $\mathrm{W}=0.91, \mathrm{P}=0.21$; Fragmentation Index, $\mathrm{W}=0.90, \mathrm{P}=0.17$; Native Richness, $\mathrm{W}=0.98, \mathrm{P}=0.98$; Exotic Richness, $\mathrm{W}=0.93, \mathrm{P}=0.44$ ). Because all data showed normal distribution, statistical analyses were conducted by Pearson correlation analyses using the software Statistica 6.0. The relationships between native and exotic species richness, fragmentation index, degradation index and altitude were assessed using the altitude where transects were sampled (Table I). The relationships between degradation index, fragmentation index and altitude were assessed using the mean altitude of fragments (average between the higher and lower altitude value per fragment) (Table I).

\section{RESULTS}

Plant SPECIES COMPOSITION

The species composition found in the study area comprised 110 species, from 95 genera and 64 families of vascular plants (Table II). Among these species, 32.7\% (36) are herbaceous, 28\% (31) trees, 18\% (20) bushes, 11\% (12) climbers, $8 \%$ (9) ferns and 1\% (2) parasitic.

Exotic and native species accounted for 15\% (16) and $85 \%$ (89) of 105 species with known origen (Table II). The best represented families of exotic species were: Poaceae with $27.7 \%$ (5 species), Asteraceae with 22.2\% (4 species), Rosaceae with 16.6\% (3 species) and Fabaceae with $16.6 \%$ (3 species). Exotic species included 72\% (13) herbaceous species, 17\% (3) shrubs and 11\% (2) trees.

The most frequent native species were Aristotelia chilensis Stuntz, Blechnum hastatum Kaulf., Luzuriaga radicans Ruiz \& Pav., Rhaphithamnus spinosus (Juss.) Moldenke, Nothofagus dombeyi and Aextoxicon punctatum, all present in more than $80 \%$ of fragments (Table II). The most frequent exotic species were Rubus constrictus Lefèvre \& P.J.Müll., Rosa rubiginosa L., Taraxacum officinale F.H.Wigg and Prunella vulgaris L., all present in more than $50 \%$ of fragments (Table II). 
RELATIONSHIPS BETWEEN ALTITUDE, FRAGMENTATION AND DEGRADATION

Patch sizes ranged from 12 to 29,642 hectares. Perimeters varied between $1,779 \mathrm{~m}$ and $127,065 \mathrm{~m}$ and the perimeter/area ratio between 4.29 and 228.51. The lowest fragmentation index was 0.012 and the highest 1.997. All fragments showed a degradation index above cero. The lowest degradation index was 0.57 and the highest 1.75 . The degradation index was significantly negatively related to mean altitude of fragments (Pearson, $\mathrm{r}=-0.64, \mathrm{P}=0.02, \mathrm{~N}=$ 12) (Fig. 2), and a positive relationship between degradation and fragmentation indexes was observed (Pearson, $r=0.75$, $\mathrm{P}=0.004, \mathrm{~N}=12$ ) (Fig. 2). Finally, fragments with lower mean altitude showed significantly greater fragmentation index (Pearson, $\mathrm{r}=-0.86, \mathrm{P}<0.001, \mathrm{~N}=12$ ) (Fig. 2).

\section{SPECIES RICHNESS PATTERNS}

Total species richness varied from 30 species in the poorest fragment to 57 in the richest. Richness of native species ranged from 27 to 48 species per fragment, while exotic species richness from 0 to 12 species per fragment. We found no significant relationship between richness of exotic and native species (Pearson, $\mathrm{r}=-0.11, \mathrm{P}=0.72, \mathrm{~N}$ $=12$ ). Exotic species richness was significantly positively related to the degradation index (Pearson, $\mathrm{r}=0.61, \mathrm{P}=$ 0.03, $\mathrm{N}=12$ ) (Fig. 3) as well as with the fragmentation index (Pearson, $\mathrm{r}=0.66, \mathrm{P}=0.02, \mathrm{~N}=12$ ) (Fig. 4). In particular, a significant positive relationship was found between exotic species richness and the density of domestic livestock faeces (Pearson, $\mathrm{r}=0.73 ; \mathrm{P}=0.01, \mathrm{~N}=12$ ). Furthermore, exotic species richness was only marginally negatively related to altitude (Pearson, $\mathrm{r}=-0.52, \mathrm{P}=0.08$, $\mathrm{N}=12$ ) (Fig. 5). In turn, native species richness was not significantly related to degradation index (Pearson, $\mathrm{r}=$ 0.19; $\mathrm{P}=0.54, \mathrm{~N}=12$ ) (Fig. 3) nor altitude (Pearson, $\mathrm{r}$ $=0.41 ; \mathrm{P}=0.18, \mathrm{~N}=12$ ) (Fig. 5), but it was marginally negatively related to the fragmentation index (Pearson, $\mathrm{r}=$ -0.54; $\mathrm{P}=0.06, \mathrm{~N}=12$ ) (Fig. 4).

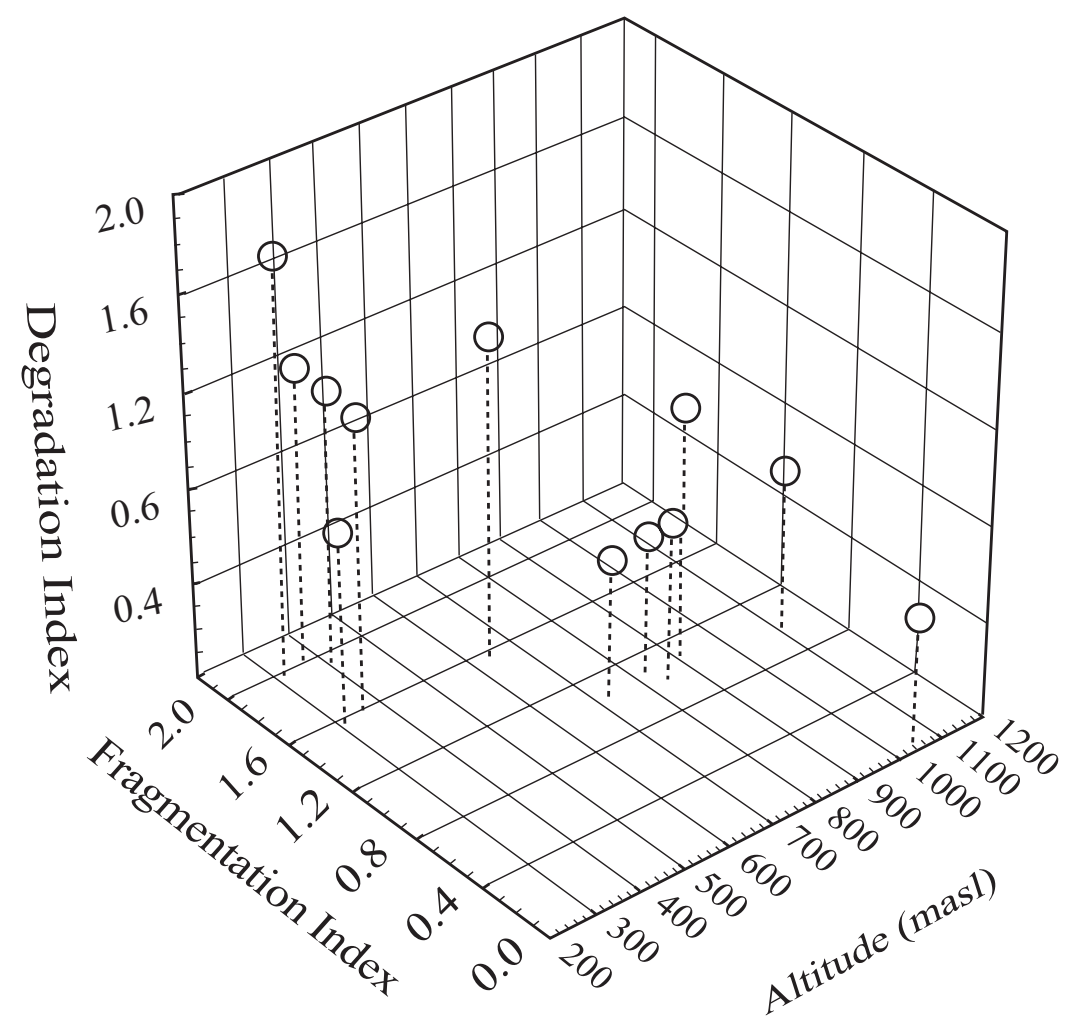

FIGURE 2. Relationship between fragmentation index, degradation index and mean altitude of fragments (average between the higher and lower levels of each fragment).

Figura 2. Relación entre índice de fragmentación, índice de degradación y altitud media de los fragmentos (promedio entre el nivel altitudinal máximo y mínimo). 
Gayana Bot. 68(2), 2011

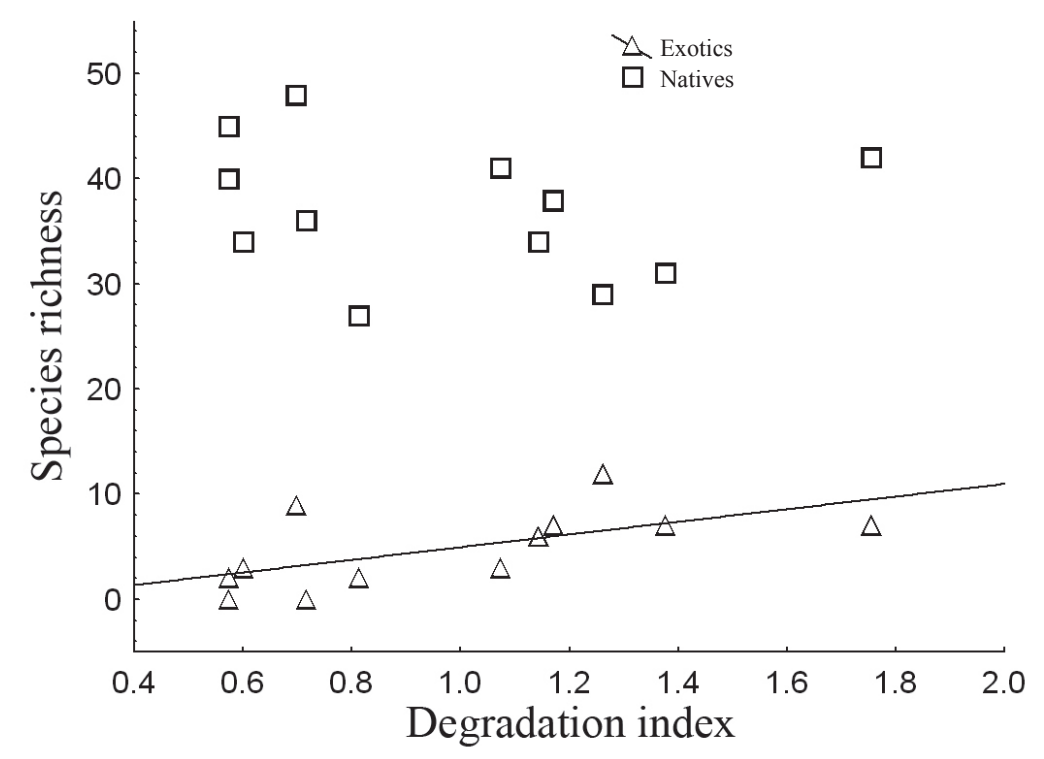

FIGURE 3. Relationship between degradation index and native and exotic species richness. Only significant $(\mathrm{P}<0.05)$ or marginally significant $(\mathrm{P}<0.1)$ relationships have a trend line (see text for results).

FIgURA 3. Relación entre índice de degradación y riqueza de especies exóticas y nativas. Sólo las relaciones significativas $(\mathrm{P}<0,05)$ o marginalmente significativas $(\mathrm{P}<0,1)$ tienen línea de tendencia (ver texto para resultados).

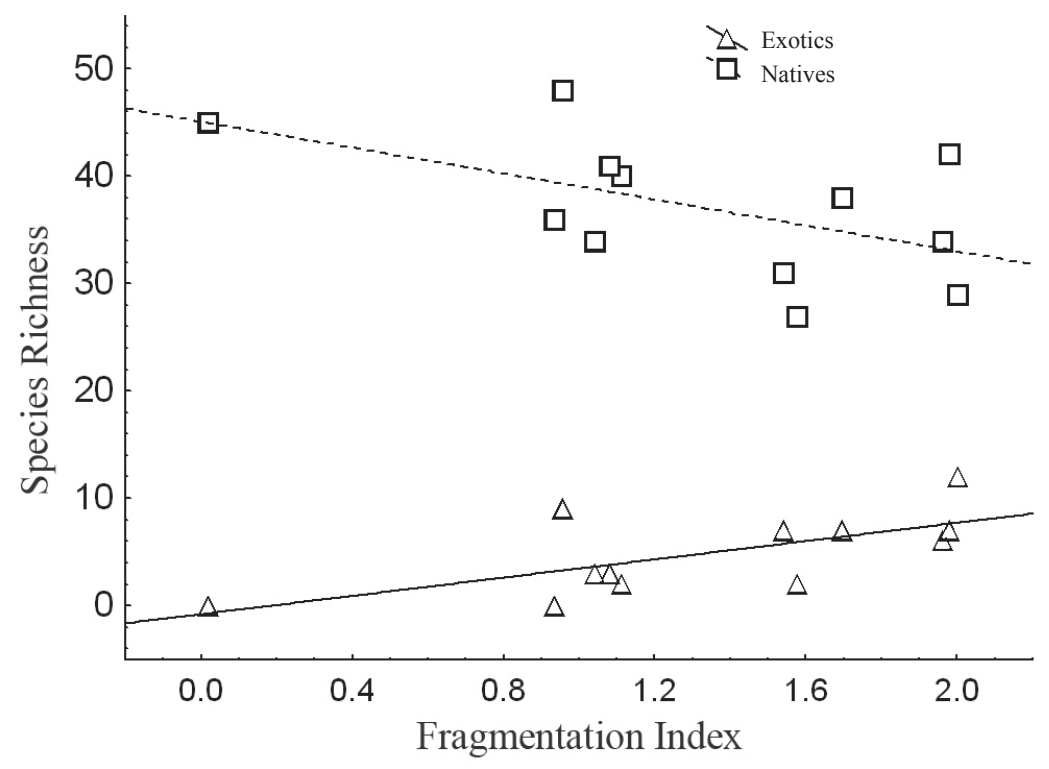

FIGURE 4. Relationship between fragmentation index and native and exotic species richness. Only significant $(\mathrm{P}<0.05)$ or marginally significant $(\mathrm{P}<0.1)$ relationships have a trend line (see text for results).

Figura 4. Relación entre índice de fragmentación y riqueza de especies exóticas y nativas. Sólo las relaciones significativas $(\mathrm{P}<0,05)$ o marginalmente significativas $(\mathrm{P}<0,1)$ tienen línea de tendencia (ver texto para resultados). 
Fragmentation, forest degradation and plant invasion: RoJAS, I. ET AL.

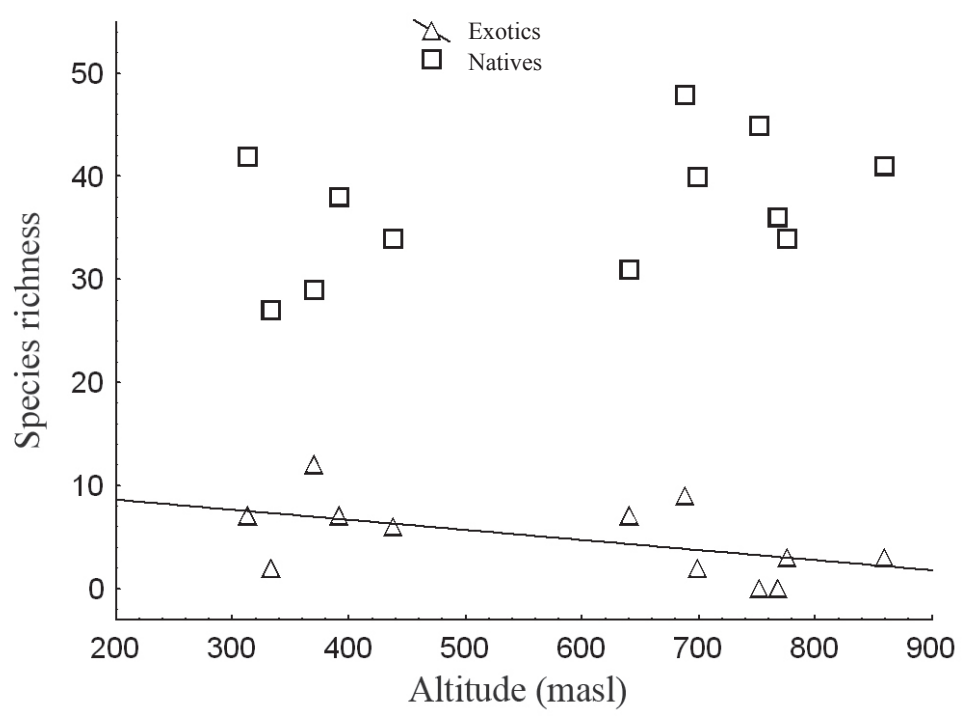

FIGURE 5. Relationship between altitude and native and exotic species richness. Only significant $(\mathrm{P}<0.05)$ or marginally significant $(\mathrm{P}<0.1)$ relationships have a trend line (see text for results).

FIgURA 5. Relación entre altitud y riqueza de especies exóticas y nativas. Sólo las relaciones significativas $(\mathrm{P}<0,05)$ o marginalmente significativas $(\mathrm{P}<0,1)$ tienen línea de tendencia (ver texto para resultados).

TABLE II. Floristic composition of the study area. Scientific name, origin (native: N, exotic: E), family, and frequency (percentage of fragments with presence) of the species are shown.

TABLA II. Composición florística del área de estudio con nombres científicos, origen geográfico de las especies (nativo: N, exótico: E), familia y frecuencia (porcentaje de fragmentos con presencia).

\begin{tabular}{|c|c|c|c|}
\hline SCIENTIFIC NAME & ORIGIN & FAMILY & $\begin{array}{c}\text { FREQUENCY } \\
(\%)\end{array}$ \\
\hline Acaena ovalifolia Ruiz \& Pav. & $\mathrm{N}$ & Rosaceae & 50.0 \\
\hline Acer pseudoplatanus L. & $\mathrm{E}$ & Aceraceae & 16.7 \\
\hline Acrisione denticulata (Hook. \& Arn.) B. Nord. & $\mathrm{N}$ & Asteraceae & 16.7 \\
\hline Adenocaulon chilense Less. & $\mathrm{N}$ & Asteraceae & 33.3 \\
\hline Adiantum chilense Kaulf. var. chilense & $\mathrm{N}$ & Adiantaceae & 50.0 \\
\hline Aextoxicon punctatum Ruiz \& Pav. & $\mathrm{N}$ & Aextoxicaceae & 83.3 \\
\hline Agrostis capillaris L. & $\mathrm{E}$ & Poaceae & 16.7 \\
\hline Alstroemeria aurea Graham & $\mathrm{N}$ & Alstroemeriaceae & 33.3 \\
\hline Amomyrtus luma (Molina) D.Legrand \& Kausel & $\mathrm{N}$ & Myrtaceae & 58.3 \\
\hline Aristotelia chilensis Stuntz & $\mathrm{N}$ & Elaeocarpaceae & 100.0 \\
\hline Asteraceae & - & Asteraceae & 8.3 \\
\hline Azara integrifolia Ruiz \& Pav. & $\mathrm{N}$ & Flacourtiaceae & 16.7 \\
\hline Azara lanceolata Hook.f. & $\mathrm{N}$ & Flacourtiaceae & 66.7 \\
\hline Azara microphylla Hook.f. & $\mathrm{N}$ & Flacourtiaceae & 41.7 \\
\hline Baccharis sphaerocephala Hook. \& Arn. & $\mathrm{N}$ & Asteraceae & 16.7 \\
\hline Berberis darwinii Hook. var. darwinii & $\mathrm{N}$ & Berberidaceae & 66.7 \\
\hline Blechnum mochaenum Kunkel & $\mathrm{N}$ & Blechnaceae & 75.0 \\
\hline
\end{tabular}


Gayana Bot. 68(2), 2011

\begin{tabular}{|c|c|c|c|}
\hline SCIENTIFIC NAME & ORIGIN & FAMILY & $\begin{array}{c}\text { FREQUENCY } \\
(\%)\end{array}$ \\
\hline Blechnum hastatum Kaulf. & $\mathrm{N}$ & Blechnaceae & 83.3 \\
\hline Blechnum magellanicum (Desv.) Mett. & $\mathrm{N}$ & Blechnaceae & 41.7 \\
\hline Blechnum penna-marina (Poir.) Kuhn & $\mathrm{N}$ & Blechnaceae & 8.3 \\
\hline Boquila trifoliata Decne. & $\mathrm{N}$ & Lardizabalaceae & 75.0 \\
\hline Caldcluvia paniculata D.Don. & $\mathrm{N}$ & Cunoniaceae & 58.3 \\
\hline Campsidium valdivianum (Phil.) Skottsb. & $\mathrm{N}$ & Bignoniaceae & 33.3 \\
\hline Cardamine aff. cordata Barnéoud & $\mathrm{N}$ & Brassicaceae & 16.7 \\
\hline Carex inconspicua Steud. & $\mathrm{N}$ & Cyperaceae & 8.3 \\
\hline Chusquea culeou E.Desv. & $\mathrm{N}$ & Poaceae & 50.0 \\
\hline Chusquea quila Kunth & $\mathrm{N}$ & Poaceae & 50.0 \\
\hline Cissus striata Ruiz \& Pav. & $\mathrm{N}$ & Vitaceae & 58.3 \\
\hline Codonorchis lessonii Lindl. & $\mathrm{N}$ & Orchidaceae & 16.7 \\
\hline Dactylis glomerata L. & $\mathrm{E}$ & Poaceae & 33.3 \\
\hline Dasyphyllum diacanthoides (Less.) Cabrera & $\mathrm{N}$ & Asteraceae & 66.7 \\
\hline Desfontainia fulgens D.Don & $\mathrm{N}$ & Desfontainiaceae & 8.3 \\
\hline Dioscorea brachybotrya Poepp. & $\mathrm{N}$ & Dioscoreaceae & 58.3 \\
\hline Diplolepis menziesii Schult. & $\mathrm{N}$ & Asclepiadaceae & 16.7 \\
\hline Drimys winteri J.R.Forst \& G. Forst. & $\mathrm{N}$ & Winteraceae & 25.0 \\
\hline Dysopsis glechomoides Müll.Arg. & $\mathrm{N}$ & Euphorbiaceae & 33.3 \\
\hline Elytropus chilensis Müll.Arg. & $\mathrm{N}$ & Apocynaceae & 16.7 \\
\hline Embothrium coccineum J.R.Forst. \& G.Forst. & $\mathrm{N}$ & Proteaceae & 33.3 \\
\hline Escallonia rosea Griseb. & $\mathrm{N}$ & Escalloniaceae & 8.3 \\
\hline Eucryphia cordifolia Cav. & $\mathrm{N}$ & Eucryphiaceae & 33.3 \\
\hline Fascicularia bicolor (Ruiz \& Pav.) Mez & $\mathrm{N}$ & Bromeliaceae & 16.7 \\
\hline Francoa appendiculata Cav. & $\mathrm{N}$ & Francoaceae & 8.3 \\
\hline Fuchsia magellanica Lam. & $\mathrm{N}$ & Onagraceae & 75.0 \\
\hline Galium hypocarpium Endl.ex Griseb. & $\mathrm{N}$ & Rubiaceae & 25.0 \\
\hline Gaultheria mucronata Phil. & $\mathrm{N}$ & Ericaceae & 33.3 \\
\hline Geranium sp. & - & Geraniaceae & 25.0 \\
\hline Gevuina avellana Molina & $\mathrm{N}$ & Proteaceae & 41.7 \\
\hline Hydrangea serratifolia F. Phil. & $\mathrm{N}$ & Hydrangeaceae & 66.7 \\
\hline Hydrocotyle poeppigii DC. & $\mathrm{N}$ & Apiaceae & 33.3 \\
\hline Hypochaeris radicata $\mathrm{L}$. & $\mathrm{E}$ & Asteraceae & 25.0 \\
\hline Hypolepis poeppigii (Kunze) R.A.Rodr. & $\mathrm{N}$ & Dennstaedtiaceae & 41.7 \\
\hline Lapageria rosea Ruiz \& Pav. & $\mathrm{N}$ & Philesiaceae & 41.7 \\
\hline Lapsana communis L. & $\mathrm{E}$ & Asteraceae & 16.7 \\
\hline Laurelia sempervirens (Ruiz \& Pav.) Tul. & $\mathrm{N}$ & Monimiaceae & 41.7 \\
\hline Laureliopsis philippiana (Looser) Schodde & $\mathrm{N}$ & Monimiaceae & 75.0 \\
\hline Lepidoceras chilense (Molina) Kuijt & $\mathrm{N}$ & Loranthaceae & 8.3 \\
\hline Loasa acanthifolia Lam. & $\mathrm{N}$ & Loasaceae & 41.7 \\
\hline
\end{tabular}


Fragmentation, forest degradation and plant invasion: ROJAS, I. ET AL.

\begin{tabular}{|c|c|c|c|}
\hline SCIENTIFIC NAME & ORIGIN & FAMILY & $\begin{array}{c}\text { FREQUENCY } \\
(\%)\end{array}$ \\
\hline Lomatia dentata $\mathrm{R} . \mathrm{Br}$. & $\mathrm{N}$ & Proteaceae & 66.7 \\
\hline Lomatia ferruginea (Cav.) R. Br. & $\mathrm{N}$ & Proteaceae & 33.3 \\
\hline Lomatia hirsuta (Lam.) Diels. ex J.F.Macbr. & $\mathrm{N}$ & Proteaceae & 58.3 \\
\hline Lophosoria quadripinnata (J.F.Gmel.) C.Chr. & $\mathrm{N}$ & Dicksoniaceae & 41.7 \\
\hline Lotus uliginosus Schkuhr & $\mathrm{E}$ & Fabaceae & 33.3 \\
\hline Luma apiculata (DC.) Burret & $\mathrm{N}$ & Myrtaceae & 75.0 \\
\hline Luzuriaga radicans Ruiz \& Pav. & $\mathrm{N}$ & Philesiaceae & 83.3 \\
\hline Lysimachia sertulata Baudo & $\mathrm{N}$ & Primulaceae & 8.3 \\
\hline Maytenus boaria Molina & $\mathrm{N}$ & Celastraceae & 25.0 \\
\hline Maytenus magellanica Hook.f. & $\mathrm{N}$ & Celastraceae & 41.7 \\
\hline Mitraria coccinea Cav. & $\mathrm{N}$ & Gesneriaceae & 66.7 \\
\hline Muehlenbeckia hastulata (Sm.) I.M.Johnst. & $\mathrm{N}$ & Polygonaceae & 41.7 \\
\hline Myoschilos oblongum Ruiz \& Pav. & $\mathrm{N}$ & Santalaceae & 25.0 \\
\hline Myrceugenia planipes O.Berg & $\mathrm{N}$ & Myrtaceae & 25.0 \\
\hline Nertera granadensis Druce & $\mathrm{N}$ & Rubiaceae & 75.0 \\
\hline Nothofagus alpina (Poepp. \& Endl.) Oerst. & $\mathrm{N}$ & Nothofagaceae & 16.7 \\
\hline Nothofagus dombeyi (Mirb.) Oerst. & $\mathrm{N}$ & Nothofagaceae & 91.7 \\
\hline Nothofagus obliqua (Mirb.) Oerst. & $\mathrm{N}$ & Nothofagaceae & 75.0 \\
\hline Osmorhiza chilensis Hook. \& Arn. & $\mathrm{N}$ & Apiaceae & 75.0 \\
\hline Ourisia ruelloides (L.f.) Kuntze & $\mathrm{N}$ & Scrophulariaceae & 33.3 \\
\hline Oxalis sp. & - & Oxalidaceae & 8.3 \\
\hline Oxalis valdiviensis Barnéoud & $\mathrm{N}$ & Oxalidaceae & 16.7 \\
\hline Persea lingue (Miers ex Bertero) Nees & $\mathrm{N}$ & Lauraceae & 41.7 \\
\hline Peumus boldus Molina & $\mathrm{N}$ & Monimiaceae & 8.3 \\
\hline Plantago lanceolata $\mathrm{L}$. & $\mathrm{E}$ & Plantaginaceae & 16.7 \\
\hline Polystichum sp. & $\mathrm{N}$ & Dryopteridaceae & 41.7 \\
\hline Prunella vulgaris L. & $\mathrm{E}$ & Labiatae & 66.7 \\
\hline Prunus sp. & $\mathrm{E}$ & Rosaceae & 33.3 \\
\hline Pteris semiadnata Phil. & $\mathrm{N}$ & Pteridaceae & 8.3 \\
\hline Raukaua laetevirens (Gay) Frodin & $\mathrm{N}$ & Araliaceae & 33.3 \\
\hline Rhamnus diffusus Clos & $\mathrm{N}$ & Rhamnaceae & 25.0 \\
\hline Rhaphithamnus spinosus (Juss.) Moldenke & $\mathrm{N}$ & Verbenaceae & 83.3 \\
\hline Ribes valdivianum Phil. & $\mathrm{N}$ & Saxifragaceae & 66.7 \\
\hline Rosa rubiginosa $\mathrm{L}$. & $\mathrm{E}$ & Rosaceae & 50.0 \\
\hline Rubus constrictus Lefèvre \& P.J.Müll. & $\mathrm{E}$ & Rosaceae & 58.3 \\
\hline Rumex acetosella $\mathrm{L}$. & $\mathrm{E}$ & Polygonaceae & 8.3 \\
\hline Saxegothaea conspicua Lindl. & $\mathrm{N}$ & Podocapaceae & 41.7 \\
\hline Solanum crispum Ruiz \& Pav. & $\mathrm{N}$ & Solanaceae & 8.3 \\
\hline Solanum cyrtopodium Dunal & $\mathrm{N}$ & Solanaceae & 41.7 \\
\hline Solanum nigrum L. & $\mathrm{E}$ & Solanaceae & 8.3 \\
\hline
\end{tabular}




\begin{tabular}{|c|c|c|c|}
\hline SCIENTIFIC NAME & ORIGIN & FAMILY & $\begin{array}{c}\text { FREQUENCY } \\
(\%)\end{array}$ \\
\hline Sophora cassioides (Phil.) Sparre & $\mathrm{N}$ & Fabaceae & 16.7 \\
\hline Taraxacum officinale G.Weber ex F.H.Wigg. & $\mathrm{E}$ & Asteraceae & 50.0 \\
\hline Trifolium pratense $\mathrm{L}$. & $\mathrm{E}$ & Fabaceae & 16.7 \\
\hline Trifolium repens $\mathrm{L}$. & $\mathrm{E}$ & Fabaceae & 8.3 \\
\hline Tristerix corymbosus (L.) Kuijt & $\mathrm{N}$ & Loranthaceae & 8.3 \\
\hline Tropaeolum speciosum Poepp. \& Endl. & $\mathrm{N}$ & Tropaeolaceae & 8.3 \\
\hline Ugni molinae Turcz. & $\mathrm{N}$ & Myrtaceae & 41.7 \\
\hline Uncinia erinacea Pers. & $\mathrm{N}$ & Cyperaceae & 50.0 \\
\hline Urtica magellanica Juss. ex. Poir. & $\mathrm{N}$ & Urticaceae & 16.7 \\
\hline Vicia sp. & - & Fabaceae & 8.3 \\
\hline Viola reichei Skottsb. ex Macloskie & $\mathrm{N}$ & Violaceae & 41.7 \\
\hline Weinmannia trichosperma Cav. & $\mathrm{N}$ & Cunoniaceae & 50.0 \\
\hline Poaceae & - & Poaceae & 16.7 \\
\hline
\end{tabular}

\section{DISCUSSION}

All the studied forest fragments, regardless the size and shape, revealed signs of some anthropic disturbance, such as selective logging, cattle and fires. Thus, the forest landscape in this locality is both fragmented and internally degraded. However, as expected, patches were not evenly degraded, with higher degradation levels observed in fragments with greater fragmentation index. In other Chilean temperate forests, more frequent fire events, cattle presence and logging have also been observed in smaller fragments (Jaña et al. 2007). Similarly, Cochrane (2001) observed a greater number of fire events in smaller fragments of tropical forests, and Hobbs (2001) more cattle presence in smaller fragments of Australian forests. Thus, the positive relationship between fragmentation and degradation seems to be a common pattern. In addition, our results suggest that human impact has mainly occurred in lowlands, which is a pattern frequently documented in fragmentation studies (Lindenmayer \& Fischer 2006).

Fragmentation has been observed to affect species richness both negatively and positively, depending on the biogeographical origin of species (Saunders et al. 1991, Tabarelli et al. 1999, Fahrig 2003, Teo et al. 2003, Lindenmayer \& Fischer 2006). Our findings support the hypothesis that fragmentation causes an increase in exotic plant invasion as we found a positive significant relationship between the fragmentation index and exotic richness. Thus, our results are consistent with many other studies about the effect of fragmentation on invasion which have generally shown a increase of exotic invasion within fragments relative to continuous or larger fragments (e.g. Harrison 1999, Hobbs 2001, Rouget et al. 2002, With 2002), and a positive edge effect (e.g. Brothers \& Spingarn 1992, Meiners \& Pickett 1999, Hobbs 2001, Bustamante et al. 2003). It is probable that increase of light in smaller fragments (Chen et al. 1995) may be enhancing exotic invasion (Saunders et al. 1991, Murcia 1995). Also, in smaller fragments interior sites (where sampling was performed) are closer to edges than the interior of larger fragments. Hence, the interior of smaller fragments is closer to the propagule source of exotic species growing in the matrix and dispersal of seeds of exotic species is more probable (e.g. Cadenasso \& Pickett 2001).

On the other hand, despite we observed only a marginally significant relationship between native species richness and fragmentation, the trend was clearly negative, similar to our hypothesis. It is probable that the low number of fragments has limited the statistical power to get a significant negative relationship. Then, this result is concordant to other studies documenting negative effects of fragmentation on native biodiversity (e.g. Saunders et al. 1991, Soulé et al. 1992, Gilliam et al. 1995, Laurance et al. 1998, Tabarelli et al. 1999, Hersperger \& Forman 2003, Lindenmayer \& Fischer 2006, Echeverría et al. 2007, Schmitt et al. 2010). As with exotic species, environmental changes produced by fragmentation (Chen et al. 1995, Murcia et al. 1995) may be detrimental for some forest-specialist native species. Therefore, our results suggest that fragmentation affects inversely to native and exotic species in this region.

Forest degradation has strong implications for habitat quality of wildlife as well as for plant species themselves (Hobbs \& Huenneke 1992, Gilliam et al. 1995, Dzwonko 
\& Loster 1997). However, we did not find a significant relationship between native species richness and degradation. It is probable that human disturbances in studied forests have produced similar environmental changes than natural disturbances commonly observed in these forest-types (e.g. small-gap dynamic) (Saldaña \& Lusk 2003, Gutiérrez et al. 2004), and hence native species are not being negatively affected by this forest degradation. By contrast, it was clear that exotic species were positively correlated with degradation, suggesting that exotic species may react faster than native ones to anthropic disturbances (e.g. Hobbs 2001, Burns 2004). The positive correlation between number of livestock faeces and exotic species richness suggests that cattle plays a role in both degrading the sites and aiding arrival/colonization of exotic species (Hobbs 2001, Brothers \& Spingarn 1992, D'Antonio 1993). Thus, herbivores may well be important facilitators of invasion in this region. Therefore, these results suggest that degradation affects in different way but not inversely to native and exotic species.

Our results suggest that the elevation range (300 to 800 masl) covered by transects had no influence on the number of native species despite we observed reduced fragmentation and degradation at higher sites. In contrast, we observed a slight although non-significant negative relationship between altitude and exotic species richness $(\mathrm{P}=0.08)$. Thus, these results may be consistent to other studies documenting a decreasing invasion with altitude mediated by a increase in fragmentation and degradation levels (e.g. Wilson et al. 1992, Schmitt et al. 2010, Alexander et al. 2011). The absence of a statistically significant correlation suggests that this may be a developing pattern while the invasion process occurs. It is unlikely that this slight trend has been produced by climatic effects of this altitudinal gradient as all fragments corresponded to the same forest type along this altitudinal gradient (Gajardo 1993). Consistent with this, we observed no significant relationship between native species richness and altitude.

Currently there is much debate about the relationship between native and exotic species (Bruno et al. 2004, Davies et al. 2005). Recent studies have demonstrated dependence on the geographic scale of this relationship (Davies et al. 2005). Generally, local scale studies have found a negative relationship between native and exotic species richness (Hobbs 2001, Davies et al. 2005), which could be explained by competition processes that are not applicable at larger scales (Hester \& Hobbs 1992, Huston 1999). However, other studies at local scale have not found significant relationships, and in some others, the relationships have been positive (Bruno et al. 2004, Chown et al. 2005). As in several other locally-based studies (e.g. Bruno et al. 2004, Chown et al. 2005), we found no significant relationship between the richness of exotic and native species. Probably, different but not inverse effects of forest degradation on exotic and native species richness contributed to this result.
In conclusion, our results suggest that the human impact in forests of this region has affected plant communities. However, specific human-caused disturbances such as fragmentation and degradation affect differently to native and exotic species, being exotics facilitated by both degradation and fragmentation and natives only negatively affected by forest fragmentation. This suggests that management of exotic species should be focused on reducing internal degradation of remnant forests as well as fragmentation and probably edge effects. In turn, in order to conservate native species management should be focused on reducing fragmentation and edge effects.

\section{ACKNOWLEDGEMENTS}

This research was supported by Darwin Initiative (Project Ref-15/006). We thank all of the field assistants, in particular R. Petitpas, F. Hernández, O. Ohrens, C. Ríos and T. Murphy. We thank landowners for granting us permission to carry out this research on their lands. The National Forestry Service CONAF gave us valuable support to conduct surveys. I. Rojas-Viada is supported by a grant from the Comisión Nacional de Investigación Científica y Tecnológica de Chile (CONICYT).

\section{BIBLIOGRAPHY}

Alexander, J., C. Kueffer, C. Daehler, P. Edwards, A. Pauchard, T. Seipel \& M. Consortium. 2011. Assembly of nonnative floras along an elevational gradients explained by directional ecological filtering. Proceeding of the National Academy of Science of the United States of America 108(2): 656-661.

Armesto, J.J., R. Rozzi, C. Smith-Ramírez \& M.T.K. Arroyo. 1998. Conservation targets in South American temperate forests. Science 282: 1271-1272.

Arroyo, M.T.K., C. Marticorena, O. Matthei \& L. Cavieres. 2000. Plant invasions in Chile: present patterns and future predictions. In: A. Mooney \& R. Hobbs (eds.), Invasive Species in a Changing World. Island Press, New York. p. $385-421$.

BECERRA, P.I. 2006. Invasión de árboles alóctonos en una cuenca pre-andina de Chile central. Gayana Botánica 63: 161174.

Brothers, T.S. \& A. Spingarn. 1992. Forest Fragmentation and Alien Plant Invasion of Central Indiana Old-Growth Forests. Conservation Biology 6: 91-100.

Bruno, J.F., C.W. Kennedy, T.A. Rand \& M.B. Grant. 2004. Landscape-scale patterns of biological invasions in shoreline plant communities. Oikos 107: 531-540.

BuRns, J.H. 2004. A comparison of invasive and non-invasive dayflowers (Commelinaceae) across experimental nutrient and water gradients. Diversity and Distributions 10: 387397.

Bustamante, R.O., I. Serey \& S.T.A. Pickett. 2003. Forest 
fragmentation, plant regeneration and invasion processes across edges in Central Chile. In: G.A. Bradshaw, P.A. Marquet (eds.), How Landscapes Change: human disturbance and ecosystem fragmentation in the Americas. Springer. p. 145-158.

Bustamante, R. \& J.A. Simonetti. 2005. Is Pinus radiata invading the native vegetation in central Chile? Demographic responses in a fragmented forest. Biological Invasions 7: 243-249.

Bustamante, R., J.A. Simonetti, A. Grez \& J. San Martín. 2005. Fragmentación y dinámica de regeneración del bosque maulino: diagnóstico actual y perspectivas futuras. En: C. Smith-Ramírez, J.J. Armesto \& C. Valdovinos (eds.), Historia, biodiversidad y ecología de los bosques costeros de Chile. Editorial Universitaria, Santiago. p. 555-564.

Cadenasso, M. \& S. Pickett. 2001. Effect of edge structure on the flux of species into forest interiors. Conservation Biology 15: 91-97.

Castro, S.A., J.A. Figueroa, M. Muñoz-Schick \& F.M. Jaksic. 2005. Minimum residence time, biogeographical origin, and life cycle as determinants of the geographical extent of naturalized plants in continental Chile. Diversity and Distributions 11: 183-191.

Ceballos, G., M.M. Vale, C. Bonacic, J. Calvo-Alvarado, R. List, N. Bynum, R. Medellín, J.A. Simonetti \& J.P. RodríGUEZ. 2009. Conservation challenges for the Austral and Neotropical America section. Conservation Biology 23: 811-7.

Chen, J., J. Franklin \& T. Spies. 1995. Growing-season microclimatic gradients from clearcut edges into oldgrowth Douglas-Fir forests. Ecological Applications 5: 74-86.

Chown, S.L., B. Hull \& K.J. Gaston. 2005. Human impacts, energy availability and invasion across Southern Ocean Islands. Global Ecology and Biogeography 14: 521-528.

Cochrane, M. 2001. Synergisms interactions between habitat fragmentation and fire evergreen tropical forest. Conservation Biology 15: 1515-1521.

D'Antonio, C.M. 1993. Mechanisms Controlling Invasion of Coastal Plant Communities by the Alien Succulent, Carpobrotus edulis. Ecology 74: 83-95.

Davies, K.F., P. Chesson, S. Harrison, B.D. Inouye, B.A. Melbourne \& K.J. Rice. 2005. Spatial heterogeneity explains the scale dependence of the native-exotic diversity relationship. Ecology 86: 1602-1610.

Di Castri, F. \& HajeK, E. 1976. Bioclimatología de Chile. Ediciones Universidad Católica de Chile. 129 pp.

Dzwonko, Z. \& S. Loster. 1997. Effects of dominant trees and anthropogenic disturbances on species richness and floristic composition of secondary communities in southern Poland. Journal of Applied Ecology 34: 861-870.

Echeverría, C., A.C. Newton, A. Lara, J.M. Rey Benayas \& D.A. Coomes. 2007. Impacts of forest fragmentation on species composition and forest structure in the temperate landscape of southern Chile. Global Ecology and Biogeography 16: 426-439.

FAHRIG, L. 2003. Effects of habitat fragmentation on biodiversity. Annual Review of Ecology and Systematics 34: 487-515.

Fischer, J. \& D.B. LindenmaYer. 2007. Landscape modification and habitat fragmentation: a sysnthesis. Global Ecology and Biogeography 16: 265-280.

Fuentes-Ramírez, A., A. Pauchard, A. Marticorena, P. Sánchez. 2010. Relación entre la invasión de Acacia dealbata Link (Fabaceae: Mimosoideae) y la riqueza de especies vegetales en el centro-sur de Chile. Gayana Botánica 67: 188-197.

Gajardo, R. 1993. La Vegetación Natural de Chile: Clasificación y Distribución Geográfica. Editorial Universitaria, Chile. $165 \mathrm{pp}$.

Gilliam, F., N. Turrill \& M. Adams. 1995. Herbaceous-layer and overstory species on clear-cut and mature central Appalachian hardwood forests. Ecological Applications 5: 947-955.

Grez, A.A., J.A. Simonetti \& R.O. Bustamante. 2006. Biodiversidad en ambientes fragmentados de Chile: patrones y procesos a diferentes escalas. Editorial Universitaria, Santiago. 229 pp.

Gutiérrez, A., J.J. Armesto \& J.C. AravenA. 2004. Disturbance and regeneration dynamics of an old-growth North Patagonian rain forest in Chiloé Island, Chile. Journal of Ecology 92: 598-608.

HARRISON, S. 1999. Local and regional diversity in a patchy landscape: native, alien and endemic herbs on serpentine. Ecology 80: 70-80.

Hersperger, A. \& R. Forman. 2003. Adjacency arrangement effects on plant diversity and composition in woodland patches. Oikos 101: 279-290.

Hester, A.J. \& R.J. Hobbs. 1992. Influence of fire and soil nutrients on native and non-native annuals at remnant vegetation edges in the West Australian wheatbelt. Journal of Vegetation Science 3: 101-108.

Hоввs, R. 2001. Synergisms among habitat fragmentation, livestock grazing, and biotic invasions in Southwestern Australia. Conservation Biology 15: 1522-1528.

Hobbs, R. \& L. Huenneke. 1992. Disturbance, diversity, and invasions: implications for conservation. Conservation Biology 6: 324-337.

Huston, M.A. 1999. Local processes and regional patterns: appropriate scales for understanding variation in the diversity of plants and animals. Oikos 86: 393-401.

Jaña, R., J.L. Celis, A. Gutiérrez, C. Cornelius \& J.J. Armesto. 2007. Diversidad en bosques fragmentados de Chiloé: ¿Son todos los fragmentos iguales? En: A. Grez, J. Simonetti \& R.O. Bustamante (eds.), Biodiversidad en ambientes fragmentados de Chile, pp. 159-190. Patrones y procesos a diferentes escalas. Editorial Universitaria, Chile.

Laurance, W., L. Ferreira, J. Rankin-De Merona, S. Laurance, R. Hutchings \& T. Lovejoy. 1998. Effects of forest fragmentation on recruitment patterns in Amazonian tree communities. Conservation Biology 12: 460-464.

Lindenmayer, D. \& J. Fischer. 2006. Habitat fragmentation and landscape change. Island Press. 328 pp.

Meiners, S. \& S. Pickett. 1999. Changes in community and population responses across a forest-field gradient. Ecography 22: 261-267.

Mooney, H.A. \& H.A. HobBs. 2000. Invasive Species in a Changing World. Island Press, California. 457 pp.

Murcia, C. 1995. Edge effects in fragmented forests: implications for conservation. Trends in Ecology and Evolution 10: 5862. 
Myers, N., R.A. Mittermeier, C.G. Mittermeier, G. Da Fonseca \& J. Kent. 2000. Biodiversity Hotspots for conservation priorities. Nature 403: 853-858.

Pauchard, A. \& P.B. Alaback. 2004. Influence of elevation, land use, and landscape context on patterns of alien plant invasions along roadsides in protected areas of SouthCentral Chile. Conservation Biology 18: 238-248.

Rouget, M., D. Richardson, S. Milton \& D. Polakow. 2002. Predicting invasion dynamics of four alien Pinus species in a highly fragmented semi-arid shrubland in South Africa. Plant Ecology 152: 79-92.

SaKai, A.K., F.W. Allendorf, J.S. Holt, D.M. Lodge, J. Molofsky, K.A. With, S. Baughman, R.J. Cabin, J.E. Cohen, N.C. Ellstrand, D.E. McCauley, P. O'Neil, I.M. Parker, J.N. Thompson \& S.G. Weller. 2001. The Population biology of invasive species. Annual Review of Ecology and Systematics 32: 305-332.

SAldañA, A. \& C.H. LuSK. 2003. Influencia de las especies del dosel en la disponibilidad de recursos y regeneración avanzada en un bosque templado lluvioso del sur de Chile. Revista Chilena de Historia Natural 76: 639-650.

Saunders, D., R. Hobbs \& C. Margules. 1991. Biological consequences of ecosystem fragmentation: A Review. Conservation Biology 5: 18-30.

Sax, D.F., J.J. Stachowicz \& S.D. Gaines. 2005. Species Invasions. Insights into ecology, evolution and biogeography. Sinauer Associates, Inc. USA. 495 pp.

Schmitt, C., M. Denich, S. Demissew, I. Fris \& H.J. Boehmer. 2010. Floristic diversity in fragmented Afromontane rainforest:
Altitudinal variation and conservation importance. Applied Vegetation Science 13: 291-304.

Soulé, M., A. Alberts \& D. Bolger. 1992. The effects of habitat fragmentation on chaparral plants and vertebrates. Oikos 63: 39-47.

Tabarelli, M., W. Mantovani \& C. Peres. 1999. Effects of habitat fragmentation on plant guild structure in the montane Atlantic forest of southeastern Brazil. Biological Conservation 91: 119-127.

Teo, D.H.L., H. Tan, R.T. Corlett, C.M. Wong \& K.Y. Shawn. 2003. Continental rain forest fragments in Singapore resist invasion by exotic plants. Journal of Biogeography 30: 305-310.

The International Plant Names Index. URL: (http://www.ipni. org/index.html). Viewed: June 23, 2011.

VÁzquEZ, D. 2002. Multiple effects of introduced mammalian herbivores in a temperate forest. Biological Invasions 4: 175-191.

Wilcove, D.S., C. McLellan \& A.P. Dobson. 1986. Habitat fragmentation in the Temperate Zone. In: M.E. Soulé (ed.), Conservation Biology: The Science of Scarcity and Diversity. Sinauer, Sunderland, Massachusetts, USA. p. 237-256.

Wilson, J.B., G.L. Rapson, M.T. Sykes, A.J. Watkins \& P.A. Williams. 1992. Distributions and climatic correlations of some exotic species along roadsides in South Island, New Zealand. Journal of Biogeography 19: 183-193.

With, K. 2002. The landscape ecology of invasive spread. Conservation Biology 16: 1192-1203

Recibido: 11.04.11

Aceptado: 05.07.11 